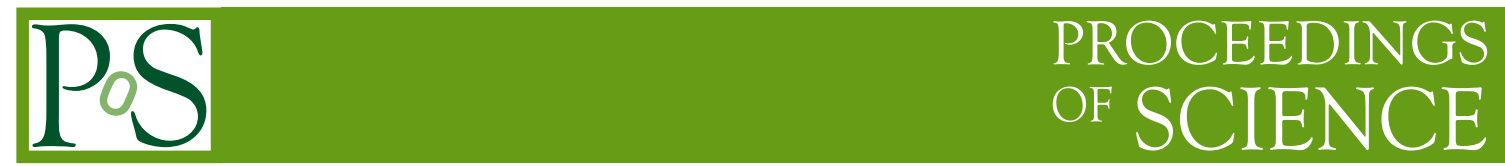

\title{
Charm at CMS
}

\author{
Jhovanny Andres Mejia Guisao ${ }^{a, *}$ \\ ${ }^{a}$ On behalf of the CMS Collaborations, \\ Instituto de Física, Universidad de Antioquia, Medellín, Colombia. \\ E-mail: jhovanny.andres.mejia.guisao@cern.ch
}

In this paper we present two studies made by the CMS Collaboration. The first is the CMS measurement of prompt $D^{*+}, D^{+}$and $D^{0}$ production cross sections at $\sqrt{s}=13 \mathrm{TeV}$. The second one corresponds to the first observation of the $B_{s}^{0} \rightarrow X(3872) \phi$ decay and the measurement of its branching fraction. The value obtained for the $B_{s}^{0}$ branching fraction is consistent with that of $B^{0}$ but smaller by a factor of two when compared with the same measurement for $B^{+}$, indicating the difference in the production dynamics of X(3872) in $B_{s}^{0}$ and $B^{+}$decays.

\footnotetext{
*** 10th International Workshop on Charm Physics (CHARM2020), ***

***31 May - 4 June, 2021 ***

*** Mexico City, Mexico - Online ***
}

\footnotetext{
${ }^{*}$ Speaker
} 


\section{Introduction}

The spectroscopy for hadrons with $b$ and $c$ quarks is an essential experimental tool to understand and study the strong interaction or QCD. For this reason, several experiments at CERN and Fermilab have included these type of studies as an important part of their programs. With the full Run 2 of the LHC, the $b$ and $c$ hadron spectroscopy at energies never reached before provides a new input to test theoretical calculations in QCD. Is worth mentioning that the gains in statistics provides us with the opportunity of looking for new and exotic decay channels.

A wide program of research in heavy flavor spectroscopy at the LHC has been performed by the CMS collaboration [1] and a number of recent results in this field are reviewed in this paper. Several other contributions in this Conference include other results not discussed here, which the reader might find interesting as well.

\section{Measurement of prompt open-charm production cross sections in proton-proton collisions at $\sqrt{s}=13 \mathrm{TeV}$}

In this analysis [2], the charm mesons are identified via their exclusive channels $p p \rightarrow$ $D^{*+} X \rightarrow D^{0} \pi_{\mathrm{s}}^{+} X \rightarrow K^{-} \pi^{+} \pi_{\mathrm{s}}^{+} X, p p \rightarrow D^{0} X \rightarrow K^{-} \pi^{+} X$, and $p p \rightarrow D^{+} X \rightarrow K^{-} \pi^{+} \pi^{+} X$, where charge conjugation is implied. The notation $\pi_{\mathrm{s}}$ indicates that given the specific kinematics of the $D^{* \pm}$ decay, this slow pion has significantly lower momentum than the kaon and pion decay products of the $D^{0}$.

The data sample used in this analysis was collected with the CMS detector at the LHC in 2016 , in proton-proton collisions at a center-of-mass energy of $\sqrt{s}=13 \mathrm{TeV}$. It corresponds to an integrated luminosity of $29 \mathrm{nb}^{-1}$, out of the $36.8 \mathrm{fb}^{-1}$ collected in the 2016 data-taking period.

The differential cross sections of the $\mathrm{D}^{* \pm}, \mathrm{D}^{ \pm}$, and $\mathrm{D}^{0}\left(\overline{\mathrm{D}}^{0}\right)$ mesons are presented in bins of transverse momentum for the first time in $b$ collisions by the CMS Collaboration. The results are also compared to several theoretical calculations and to previous measurements.

The charm meson differential cross section $\mathrm{d} \sigma / \mathrm{d} p_{T}$ is measured using $\mathrm{p}_{T}$ between 4 and 100 $\mathrm{GeV}$ in 9 bins in the pseudorapidity range $|\eta|<2.1$. The signal yields are determined using unbinned maximum-likelihood fits to the invariant mass distributions for the various decay modes (the $\Delta M$ distribution is considered for the $D^{*+}$ ) in each $\mathrm{p}_{T}$ bin. The signal components are modelled by the sum of two Gaussian functions to account for the non-uniform resolution over the detector, keeping the mean, width, and normalization term as free parameters. The combinatorial background is described with different functions, according to the decay mode.

The aim of this work is to measure the prompt open-charm production cross sections. Thus, the non-prompt contribution, such as charm mesons coming from $b$ hadron decays, has been evaluated using a simulated event sample. The contamination rate resulted to be between 5 and $17 \%$ in the different $\mathrm{p}_{T}$ bins and it has been subtracted to the total visible cross section measured using experimental data.

Several systematic uncertainty sources are considered in the measurement of the charm meson cross sections. The dominant effects come from the uncertainty related to the tracking efficiency and the mass distribution modelling used in the fit for both signal and background components. 
The differential cross sections for prompt charm meson production as a function of $\mathrm{p}_{T}$ are determined using the equations:

$$
\frac{\mathrm{d} \sigma(p p \rightarrow D X)}{\mathrm{d} p_{T}}=\frac{N_{i}(D \rightarrow f)}{\Delta p_{T i} \mathcal{B}(D \rightarrow f) \mathcal{L} \varepsilon_{i, t o t}(D \rightarrow f)},
$$

where $N_{i}(D \rightarrow f)$ is the number of prompt charm mesons reconstructed in the selected final state (including the charge conjugate final state) for each bin i, after correcting for contamination; $\Delta p_{T}$ is the $p_{T}$ bin width, $\mathbb{B}(D \rightarrow f)$ is the branching fraction of the reconstructed final state, $\varepsilon_{i, t o t}(D \rightarrow f)$ is the total reconstruction efficiency of the decay chain evaluated in the simulated events sample, and $\mathcal{L}$ is the collected luminosity.

The differential cross sections for the three mesons as a function of $p_{T}$ are shown in Fig. 1, where the data points (black) are compared to several Monte Carlo event models and theoretical predictions (horizontal lines and bands). The vertical lines on the data points show the statistical and systematic uncertainties added in quadrature.
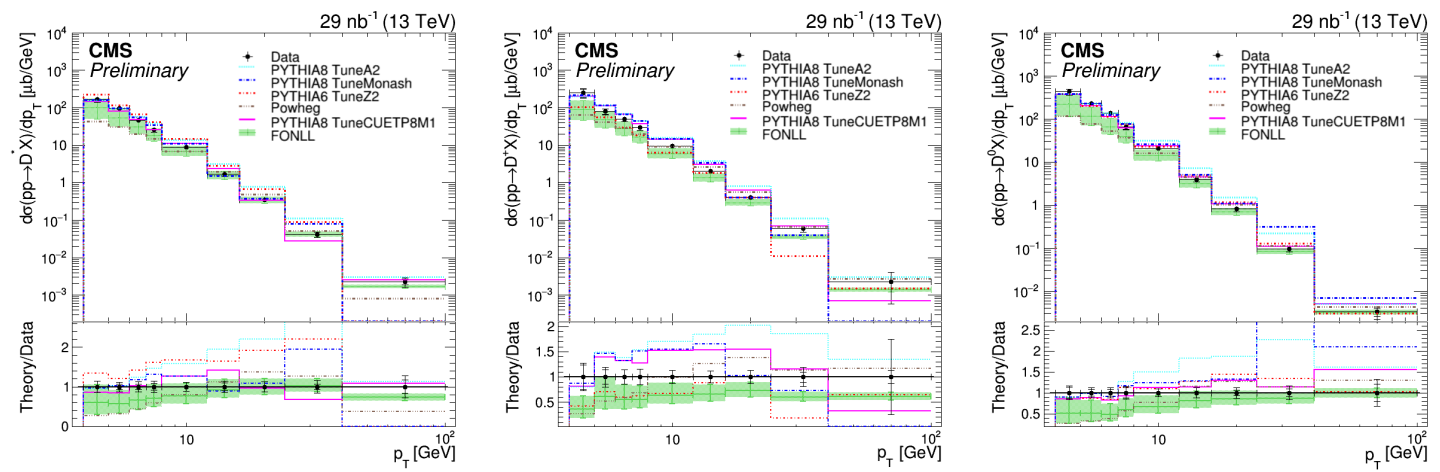

Figure 1: Differential cross sections $d \sigma / d p_{T}$ for the $\mathrm{D}^{*+}$ (left), $\mathrm{D}^{+}$(middle), and $\mathrm{D}^{0}$ (right) mesons. Data points (black) are compared with several MC simulation models and theoretical predictions. The statistical and total uncertainties are reported separated by horizontal bars. On the lower panel of the figures, the ratio of the predictions to the central value of the data is shown [2].

The agreement with the different predictions is fair in the wide kinematic range analyzed. None of the MC event generator models describes the data well in all the observed regions. Overall, the best description of the data is given by the upper edge of the FONLL uncertainty band.

For these proceedings we have made a selection of results from [2]. Indeed, here we only show a comparison between the CMS data and some previous measurements obtained in serveral LHC experiments in order to study the evolution of the cross section with respect to different kinematic regions and as a function of the center of mass energy.

With that in mind, we studied measurements from the ATLAS experiment performed at $\sqrt{s}=$ $7 \mathrm{TeV}$, the closest in terms of acceptance and kinematic range to our analysis [3]. Indeed, in Fig. 2 (left) both the ATLAS and the CMS results are shown, compared to the respective FONLL predictions at $\sqrt{s}=13 \mathrm{TeV}$ and $7 \mathrm{TeV}$ (left). Since the ATLAS results include both prompt and nonprompt charm mesons, the corresponding FONLL predictions include these components as well. The results are in good agreement in terms of shape and the comparison between the data points and the theory predictions is very similar for both experiments. The central value of the 
FONLL predictions tends to underestimate the data, but the upper edge of the FONLL bands agrees reasonably well.

A comparison with results from ALICE [4] for the cross sections at $\sqrt{s}=7 \mathrm{TeV}$ in the range $1<p_{T}<24 \mathrm{GeV}$ and for the rapidity region $|y|<0.5$ is shown in Fig. 2 (right). It is important to take to remind the reader that the cross section definition by ALICE differs by a factor of two from that of the CMS Collaboration, since in the former the charged conjugates are not included. The same is true for the corresponding FONLL predictions as well. To provide a relevant comparison, the CMS data are also given for $p_{T}<24 \mathrm{GeV}$. Both sets of data are consistent with the respective FONLL predictions.
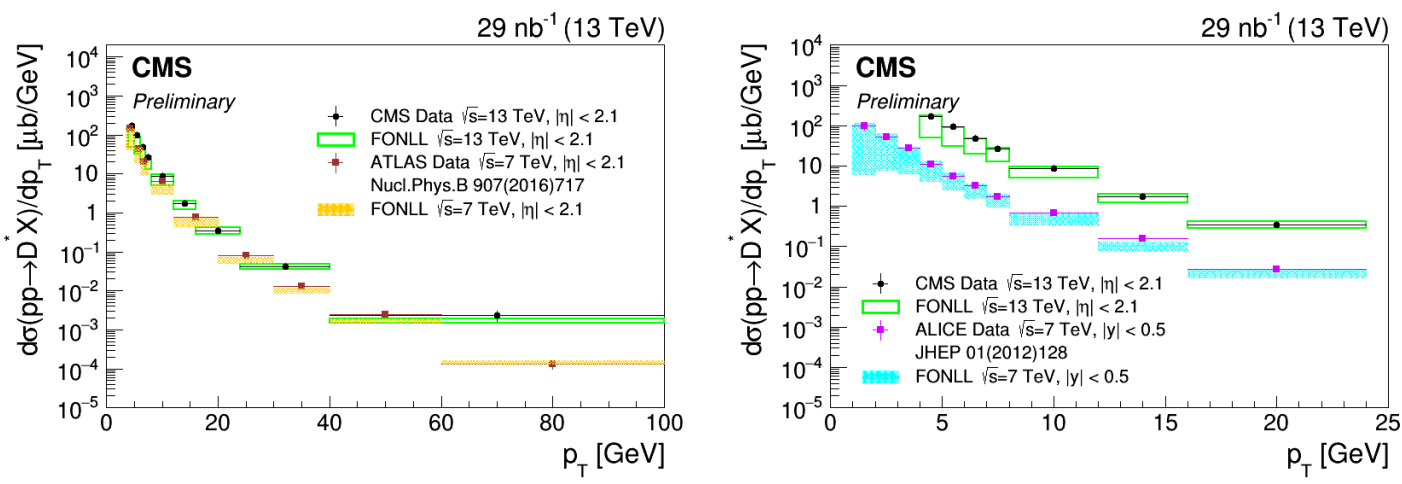

Figure 2: On the left: differential cross section $d \sigma / d p_{T}$ for the $D^{*+}$ meson, comparing the CMS (circle) and ATLAS (square) data points to the respective FONLL predictions at $\sqrt{s}=13 \mathrm{TeV}$ (empty box) and $\sqrt{s}=$ $7 \mathrm{TeV}$ (filled box).The corresponding FONLL predictions include both prompt and nonprompt charm meson cross section components as in the ATLAS data. On the right we find the differential cross section $d \sigma / d p_{T}$ for the $D^{*+}$ mesons. Compare the CMS (circle) and ALICE (square) data points with the respective FONLL predictions at $\sqrt{s}=13 \mathrm{TeV}$ (empty box) and $\sqrt{s}=7 \mathrm{TeV}$ (filled box). The cross section definition taken by ALICE and CMS differs by a factor two, given that in ALICE the charged conjugates are not included. The same is true for the corresponding FONLL predictions [2].

\section{Observation of the $B_{s}^{0} \rightarrow X(3872) \phi$ decay}

The discovery of $X(3872)$ opened a new era of exotic, quarkonium-like spectroscopy. Many new states with unusual properties have been observed, including several charged states. Prompt processes were found to be the dominant $X(3872)$ production mechanism in hadron colliders. The nature of $X(3872)$ remains unexplained. Important information about the $X(3872)$ production in weak decays can be extracted by comparing the branching fractions $\mathcal{B}(B \rightarrow X(3872) h)$ for different $\mathrm{B}$ mesons, where $h$ denotes a light hadron. More measurements of B hadron decays involving $X(3872)$ production would provide important inputs for understanding its internal structure and creation dynamics.

The CMS Collaboration has recently [5] observed a new decay mode involving the $X$ (3872) reconstructed by its decay to $J / \psi \pi^{+} \pi^{-}$. Using $p p$ collision data collected at $\sqrt{13} \mathrm{TeV}$ in 2016-2018, for an integrated luminosity of about $140 \mathrm{fb}^{-1}$, the signal of $B_{s}^{0} \rightarrow X(3872) \phi$ decay is extracted with reference to the control channel $B_{S}^{0} \rightarrow \psi(2 S) \phi$, used as normalization decay for the branching 
fraction measurement, since its identical final-state particles have very similar decay topology and kinematics.

The signal is extracted using a two-dimensional (2D) fit to $M\left(J / \psi \pi^{+} \pi^{-}\right)$and $M\left(K^{+} K^{-}\right)$, after requiring $M\left(J / \psi \pi^{+} \pi^{-} K^{+} K^{-}\right)$to be in a narrow interval around the known $B_{s}^{0}$ meson mass. The measured $J / \psi \pi^{+} \pi^{-}$invariant mass distribution is shown in Fig. 3 (left). The signal yield is determined to be $299 \pm 39$ and allows us to estimate the statistical significance of more than 6 standard deviations once systematic uncertainties have been included. This represents the first observation of the $B_{s}^{0} \rightarrow X(3872) \phi$ decay. The evaluation of the residual non- $B_{s}^{0}$ background is determined by using the non-resonant background-subtracted $M(X(3872) \phi)$ obtained by means of the ${ }_{s} \mathscr{P}$ lot technique [6]; the residual background contribution is estimated at the $1.7 \%$ level $(0.5 \%$ in the $\psi(2 S) \phi$ case). The invariant $J / \psi \pi^{+} \pi^{-} K^{+} K^{-}$mass distribution is shown in Fig. 3 (right).
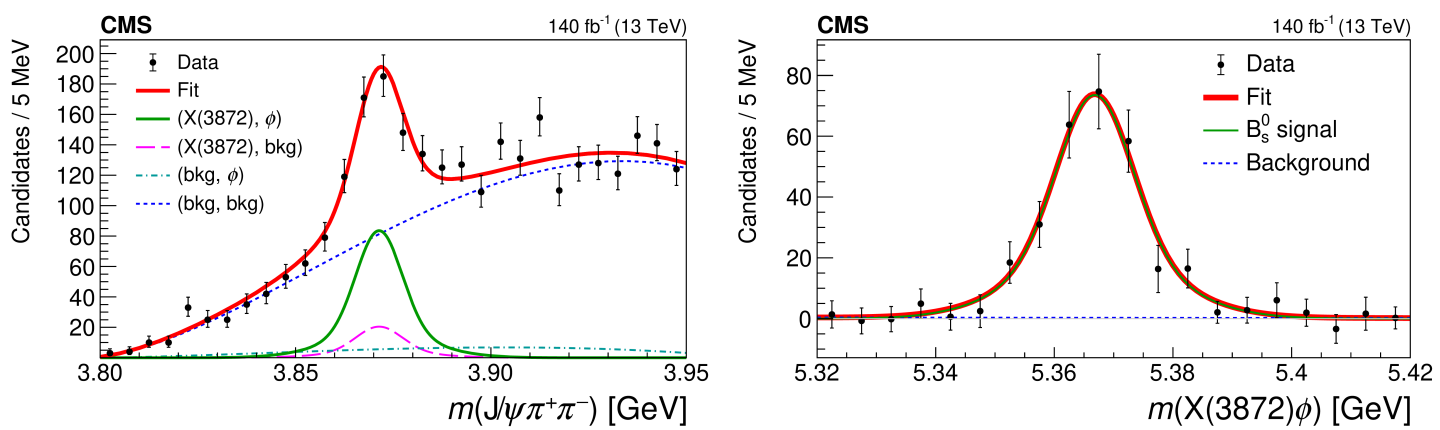

Figure 3: On the left: $J / \psi \pi^{+} \pi^{-}$invariant mass distribution in the selected $B_{s}^{0} \rightarrow X(3872) \phi$ candidates in data [5]. On the right: $X(3872) \phi$ invariant mass distribution after subtraction of non- $\phi K^{+} K^{-}$combinations and non- $X(3872) J / \psi \pi^{+} \pi^{-}$combinations [5].

Using a similar procedure the yield $15359 \pm 171$ was found for the normalization $B_{s}^{0} \rightarrow \psi(2 S) \phi$ decay channel. Correcting for the efficiency ratios, and combined with the $B_{s}^{0} \rightarrow X(3872) \phi$ yield, we obtain a value

$$
\mathscr{R}=\frac{\mathscr{B}\left(B_{s}^{0} \rightarrow X(3872) \phi\right) \mathscr{B}\left(X(3872) \rightarrow J / \psi \pi^{+} \pi^{-}\right)}{\mathscr{B}\left(B_{s}^{0} \rightarrow \psi(2 S) \phi\right) \mathscr{B}\left(\psi(2 S) \rightarrow J / \psi \pi^{+} \pi^{-}\right)}=(2.21 \pm 0.29(\text { stat }) \pm 0.17(\text { syst })) . \%
$$

By multiplying the measured ratio $\mathscr{R}$ by the known denominator branching fractions, taken from PDG [7] one obtains

$\mathscr{B}\left(B_{s}^{0} \rightarrow X(3872) \phi\right) \mathscr{B}\left(X(3872) \rightarrow J / \psi \pi^{+} \pi^{-}\right)=(4.14 \pm 0.54($ stat $) \pm 0.32($ syst $) \pm 0.46(\mathscr{B})) \times 10^{-6}$.

This product can be compared to similar results of branching fractions for the $B^{+} \rightarrow X(3872) K^{+}$ and $B^{0} \rightarrow X(3872) K^{(*) 0}$ decays in Fig. 4. The measured value for $B_{S}^{0}$ is consistent with that for $B^{0}$ but about twice smaller than that of the $B^{+}$.

These results may help understanding the nature of $X(3872)$ since they seem to suggest a difference in the production dynamics of the $X(3872)$ in both $B^{0}$ and $B_{s}^{0}$ when compared to the $B^{+}$ decay, with respect to the conventional $\psi(2 S)$, for which the neutral and charged B meson decays 


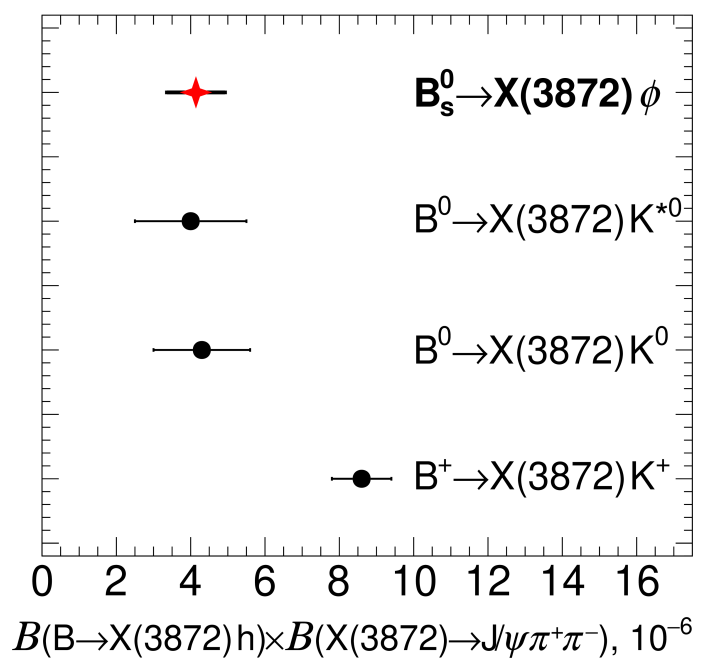

Figure 4: Comparison of the branching fraction products $\mathcal{B}\left(B_{s}^{0} \rightarrow X(3872) h\right) \times \mathscr{B}\left(X(3872) \rightarrow J / \psi \pi^{+} \pi^{-}\right)$ for $B^{+}, B^{0}$, and $B_{s}^{0}$ decays [7], where the last CMS result is highlighted in red [8].

show compatible branching fractions. An explanation for the observed difference in the decay branching fractions has been proposed [9] within the tetraquark model of the $X(3872)$ state.

\section{Acknowledgements}

A special note of acknowledgement for the support from Ministerio de Ciencia, Tecnología e Innovación (Colombia) and Universidad de Antioquia. We would like to thank the CHARM2020 conference organizers for the excellent program and the opportunity to present these results.

\section{References}

[1] CMS Collaboration, "The CMS Experiment at the CERN LHC", JINST 3, S08004 (2008).

[2] CMS Collaboration, "Measurement of prompt open-charm production cross sections in protonproton collisions at $\sqrt{13}$ TeV", CMS-PAS-BPH-18-003, http://cds.cern.ch/record/ 2720606.

[3] ATLAS Collaboration, "Measurement of $D^{* \pm}, D^{ \pm}$and $D_{s}^{+ \pm}$meson production cross sections in pp collisions at $\sqrt{7} \mathrm{TeV}$ with the ATLAS detector", Nucl. Phys. B 907 (2016) 717.

[4] ALICE Collaboration, "Measurement of charm production at central rapidity in proton-proton collisions at $\sqrt{7} \mathrm{TeV} "$, JHEP 01 (2012) 128.

[5] CMS Collaboration, "Observation of the $B_{s}^{0} \rightarrow X(3872) \phi$ decay", Phys. Rev. Lett. 125, 152001.

[6] M. Pivk and F. R. Le Diberder, "s $\mathscr{P}$ lot: A statistical tool to unfold data distributions", Nucl. Instrum. Methods Phys. Res., Sect. A 555, 356 (2005). 
[7] M. Tanabashi et al. (Particle Data Group), Review of particle physics, Phys. Rev. D 98, 030001 (2018).

[8] https://cms-results.web.cern.ch/cms-results/public-results/ publications/BPH-17-005/index.html.

[9] L. Maiani, A. D. Polosa, and V. Riquer, " The $X(3872)$ tetraquarks in B and $B_{s}^{0}$ decays", Phys. Rev. D 102, 034017 (2020). 